Supplement of Hydrol. Earth Syst. Sci., 22, 5847-5865, 2018

https://doi.org/10.5194/hess-22-5847-2018-supplement

(C) Author(s) 2018. This work is distributed under

the Creative Commons Attribution 4.0 License.

(c) (1)

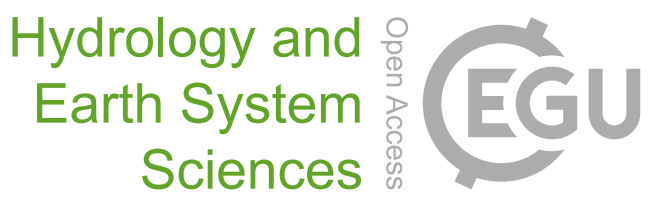

Supplement of

\title{
Studying catchment storm response using event- and pre-event-water volumes as fractions of precipitation rather than discharge
}

Jana von Freyberg et al.

Correspondence to: Jana von Freyberg (jana.vonfreyberg@usys.ethz.ch)

The copyright of individual parts of the supplement might differ from the CC BY 4.0 License. 
- Table S1 with results of the hydrograph separation analysis based on $\delta^{18} \mathrm{O}$

- Figs. S1-S4: Time series of the 24 storm events, showing precipitation hyetographs (top panel), deuterium abundance $\left(\delta^{2} \mathrm{H}\right)$ in precipitation (with individual measurements in dark blue and incremental weighted means in light blue), and deuterium streamwater (black, second panel from top), discharge hydrograph separated into event and pre-event water (third panel from the top), event water fraction of discharge (error bars indicate $\pm 1 S E$, light grey circles indicate linearly interpolated event water fractions when discharge isotope measurements are missing (second panel from the bottom), as well as soil moisture at the grassland site (light green) and forest site (dark green, no data in 2016; bottom panel 
Table S 1: Results of the hydrograph separation analysis based on $\delta^{18} O$. Columns are total discharge $(Q)$, total precipitation $(P)$, the runoff coefficient $(Q / P)$, event and pre-event discharge as whole-storm totals $\left(Q_{\mathrm{e}}\right.$ and $\left.Q_{\mathrm{pe}}\right)$, the maximum instantaneous event water fraction $q_{\mathrm{e}, i} / q_{\mathrm{i}}$ and its value at peak flow, the event water fractions of discharge and precipitation $\left(Q_{\mathrm{e}} / Q\right.$ and $\left.Q_{\mathrm{e}} / P\right)$, and preevent discharge as a fraction of precipitation $\left(Q_{\mathrm{pe}} / P\right)$.

\begin{tabular}{|c|c|c|c|c|c|c|c|c|c|c|}
\hline Event & $\underset{(\mathrm{mm})}{Q}$ & $\begin{array}{c}P \\
(\mathrm{~mm})\end{array}$ & $\underset{(-)}{Q / P}$ & $Q_{\mathrm{e}} \pm S E(\mathrm{~mm})$ & $\begin{array}{l}Q_{\mathrm{pe} \pm S E} \pm S \\
(\mathrm{~mm})\end{array}$ & $\begin{array}{c}\max \left(q_{\mathrm{e}, i} / q_{\mathrm{i}}\right) \pm \\
S E(-)\end{array}$ & $\begin{array}{c}q_{\mathrm{e}, \mathrm{i}} / q_{\mathrm{i}} \text { at peak } \\
\text { flow } \pm S E(-)\end{array}$ & $Q_{\mathrm{e}} / Q \leq S E(-)$ & $Q_{\mathrm{e}} / P \pm S E(-)$ & $Q_{\mathrm{pe}} / P \pm S E(-)$ \\
\hline 17Sep2016 & 0.8 & 9.2 & 0.08 & - a) & - & - & - & - & - & - \\
\hline 02 Oct 2016 & 4.8 & 21.6 & 0.22 & $1.16 \pm 0.07$ & $3.63 \pm 0.07$ & $0.324 \pm 0.036$ & $0.323 \pm 0.035$ & $0.243 \pm 0.015$ & $0.054 \pm 0.003$ & $0.168 \pm 0.003$ \\
\hline 09 Oct 2016 & 0.4 & 12.6 & 0.03 & $0.03 \pm 0.00$ & $0.41 \pm 0.00$ & $0.096 \pm 0.018$ & $0.059 \pm 0.018$ & $0.066 \pm 0.003$ & $0.002 \pm 0.000$ & $0.033 \pm 0.000$ \\
\hline 13May2017 & 9.5 & 20.8 & 0.46 & $1.47 \pm 0.21$ & $7.09 \pm 0.21$ & $0.321 \pm 0.079$ & $0.226 \pm 0.076$ & $0.172 \pm 0.024$ & $0.071 \pm 0.010$ & $0.341 \pm 0.010$ \\
\hline 19May2017 & 5.3 & 17.6 & 0.30 & $0.12 \pm 0.03$ & $3.02 \pm 0.03$ & $0.090 \pm 0.044$ & $0.049 \pm 0.033$ & $0.039 \pm 0.009$ & $0.007 \pm 0.002$ & $0.171 \pm 0.002$ \\
\hline 01Jun2017 & 7.5 & 28.5 & 0.26 & $1.77 \pm 0.15$ & $5.56 \pm 0.15$ & $0.438 \pm 0.053$ & $0.287 \pm 0.049$ & $0.241 \pm 0.021$ & $0.062 \pm 0.005$ & $0.195 \pm 0.005$ \\
\hline 04Jun2017 & 17.4 & 33.7 & 0.52 & $2.65 \pm 0.32$ & $13.96 \pm 0.32$ & $0.189 \pm 0.059$ & $0.166 \pm 0.054$ & $0.159 \pm 0.020$ & $0.079 \pm 0.010$ & $0.414 \pm 0.010$ \\
\hline 07Jun2017 & 8.0 & 11.2 & 0.72 & $0.37 \pm 0.04$ & $7.68 \pm 0.04$ & $0.105 \pm 0.028$ & $0.068 \pm 0.028$ & $0.046 \pm 0.005$ & $0.033 \pm 0.004$ & $0.685 \pm 0.004$ \\
\hline 16Jun2017 & 17.1 & 46.0 & 0.37 & $7.03 \pm 0.75$ & $10.04 \pm 0.75$ & $0.765 \pm 0.165$ & $0.453 \pm 0.078$ & $0.412 \pm 0.044$ & $0.153 \pm 0.016$ & $0.218 \pm 0.016$ \\
\hline 25Jun2017 & 1.7 & 21.2 & 0.08 & $0.27 \pm 0.01$ & $1.45 \pm 0.01$ & $0.205 \pm 0.024$ & $0.205 \pm 0.024$ & $0.159 \pm 0.007$ & $0.013 \pm 0.001$ & $0.068 \pm 0.001$ \\
\hline 10Jul2017 & 7.0 & 25.4 & 0.28 & $3.83 \pm 0.40$ & $2.99 \pm 0.40$ & $0.645 \pm 0.103$ & $0.643 \pm 0.094$ & $0.562 \pm 0.059$ & $0.151 \pm 0.016$ & $0.118 \pm 0.016$ \\
\hline 19Jul2017 & 4.0 & 20.2 & 0.20 & $1.07 \pm 0.04$ & $2.99 \pm 0.04$ & $0.333 \pm 0.031$ & $0.331 \pm 0.030$ & $0.263 \pm 0.010$ & $0.053 \pm 0.002$ & $0.148 \pm 0.002$ \\
\hline 27Jul2017 & 7.7 & 12.9 & 0.59 & $0.62 \pm 0.03$ & $7.05 \pm 0.03$ & $0.134 \pm 0.022$ & $0.108 \pm 0.020$ & $0.081 \pm 0.004$ & $0.048 \pm 0.002$ & $0.547 \pm 0.002$ \\
\hline 05Aug2017 & 2.7 & 17.1 & 0.16 & $0.51 \pm 0.03$ & $2.15 \pm 0.03$ & $0.246 \pm 0.046$ & $0.227 \pm 0.047$ & $0.193 \pm 0.012$ & $0.030 \pm 0.002$ & $0.126 \pm 0.002$ \\
\hline 06Aug2017 & 4.8 & 11.2 & 0.43 & $0.54 \pm 0.04$ & $4.24 \pm 0.04$ & $0.170 \pm 0.034$ & $0.149 \pm 0.034$ & $0.112 \pm 0.008$ & $0.048 \pm 0.003$ & $0.379 \pm 0.003$ \\
\hline 15Aug2017 & 2.3 & 8.2 & 0.28 & $0.09 \pm 0.01$ & $1.91 \pm 0.01$ & $0.073 \pm 0.021$ & $0.057 \pm 0.022$ & $0.047 \pm 0.003$ & $0.011 \pm 0.001$ & $0.233 \pm 0.001$ \\
\hline 18Aug2017 & 25.6 & 56.3 & 0.45 & $16.39 \pm 1.16$ & $9.55 \pm 1.16$ & $0.697 \pm 0.078$ & $0.682 \pm 0.067$ & $0.632 \pm 0.045$ & $0.291 \pm 0.021$ & $0.170 \pm 0.021$ \\
\hline 12Sep2017 & 9.2 & 19.7 & 0.46 & $0.30 \pm 0.07$ & $8.86 \pm 0.07$ & $0.140 \pm 0.039$ & $0.013 \pm 0.047$ & $0.032 \pm 0.007$ & $0.015 \pm 0.003$ & $0.450 \pm 0.003$ \\
\hline 25Sep2017 & 3.5 & 15.2 & 0.23 & $0.62 \pm 0.04$ & $2.92 \pm 0.04$ & $0.331 \pm 0.034$ & $0.227 \pm 0.036$ & $0.175 \pm 0.011$ & $0.041 \pm 0.003$ & $0.192 \pm 0.003$ \\
\hline 02 Oct 2017 & 20.9 & 39.1 & 0.53 & $4.99 \pm 0.69$ & $15.79 \pm 0.69$ & $0.286 \pm 0.076$ & $0.274 \pm 0.063$ & $0.240 \pm 0.033$ & $0.128 \pm 0.018$ & $0.404 \pm 0.018$ \\
\hline 05 Oct 2017 & 20.5 & 33.5 & 0.61 & $5.33 \pm 0.26$ & $15.34 \pm 0.26$ & $0.332 \pm 0.035$ & $0.314 \pm 0.033$ & $0.258 \pm 0.012$ & $0.159 \pm 0.008$ & $0.458 \pm 0.008$ \\
\hline $22 \mathrm{Oct} 2017$ & 25.5 & 63.2 & 0.40 & $12.60 \pm 0.89$ & $12.62 \pm 0.89$ & $0.737 \pm 0.063$ & $0.683 \pm 0.064$ & $0.499 \pm 0.035$ & $0.199 \pm 0.014$ & $0.200 \pm 0.014$ \\
\hline 26 Oct 2017 & 4.3 & 12.5 & 0.34 & $0.62 \pm 0.07$ & $3.21 \pm 0.07$ & $0.212 \pm 0.054$ & $0.187 \pm 0.054$ & $0.161 \pm 0.018$ & $0.049 \pm 0.005$ & $0.257 \pm 0.005$ \\
\hline 29 Oct 2017 & 25.7 & 44.8 & 0.57 & $8.02 \pm 0.36$ & $17.57 \pm 0.36$ & $0.376 \pm 0.036$ & $0.365 \pm 0.036$ & $0.313 \pm 0.014$ & $0.179 \pm 0.008$ & $0.392 \pm 0.008$ \\
\hline
\end{tabular}

a) Unrealistic results were obtained for storm event 17Sep2017 when $\delta^{18} \mathrm{O}$ was used as a tracer. 

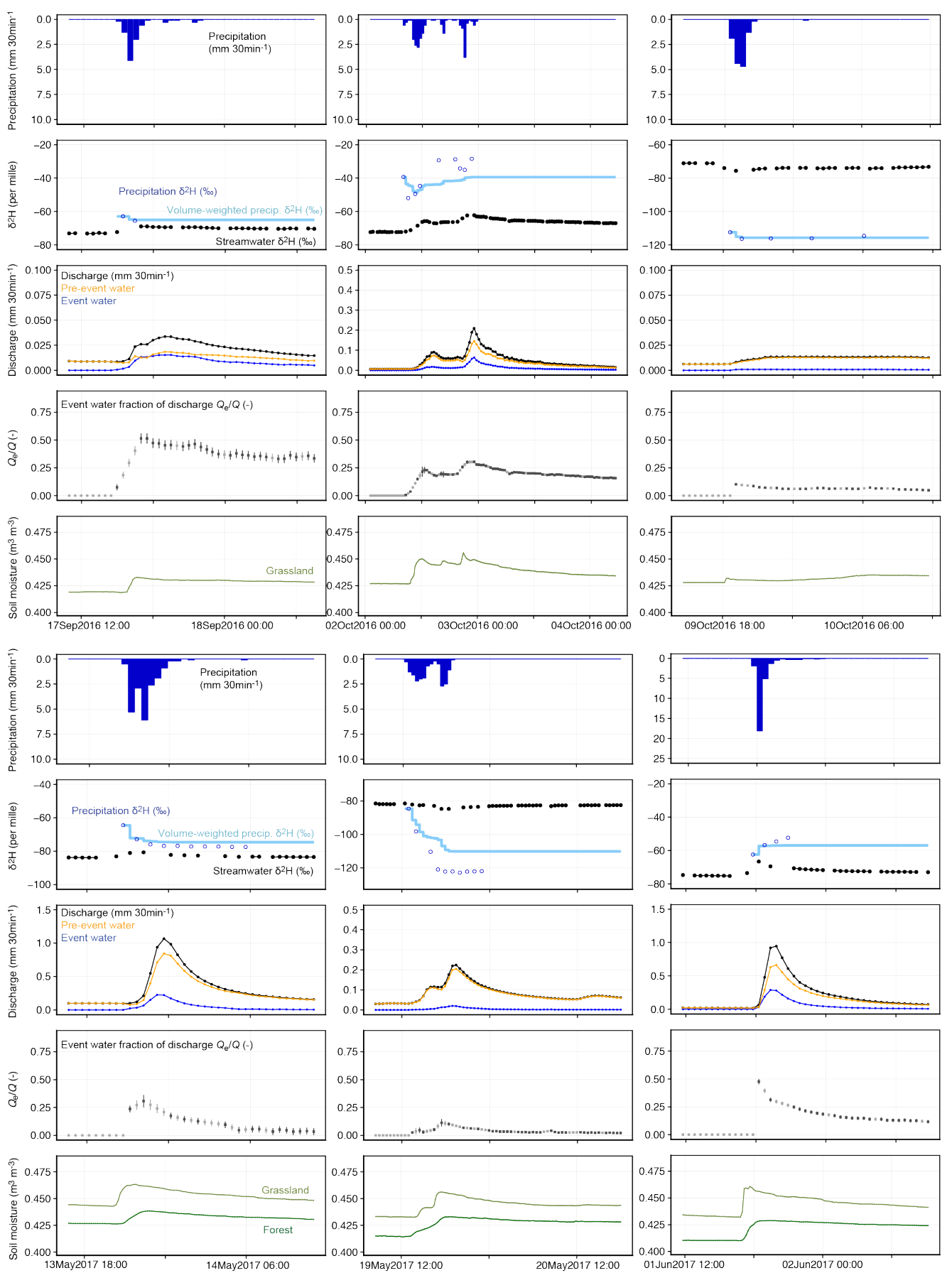

Figure S 1: Time series of storm events between 17Sep2016 and 01Jun2017, showing precipitation hyetographs (top panel), deuterium abundance $\left(\delta^{2} \mathrm{H}\right)$ in precipitation (with individual measurements in dark blue and incremental weighted means in light blue), and deuterium streamwater (black, second panel from top), discharge hydrograph separated into event and pre-event water (third panel from the top), event water fraction of discharge (error bars indicate $\pm 1 S E$, light grey circles indicate linearly interpolated event water fractions when discharge isotope measurements are missing (second panel from the bottom), as well as soil moisture at the grassland site (light green) and forest site (dark green, no data in 2016; bottom panel). 

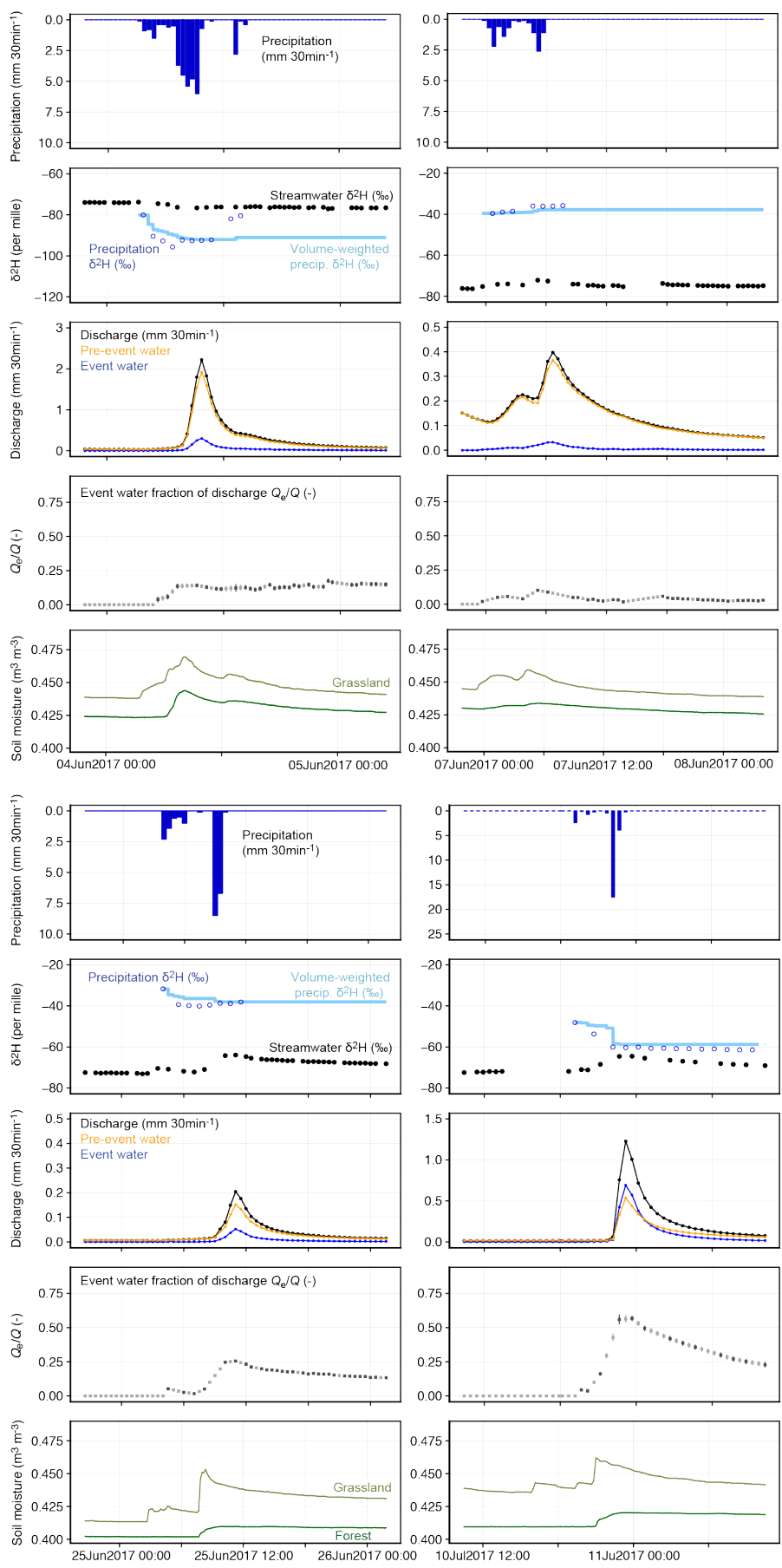

Figure S 2: Time series of storm events between 04Jun2017and 20Jul2017, showing precipitation hyetographs (top panel), deuterium abundance $\left(\delta^{2} \mathbf{H}\right)$ in precipitation (with individual measurements in dark blue and incremental weighted means in light blue), and deuterium streamwater (black, second panel from top), discharge hydrograph separated into event and pre-event water

(third panel from the top), event water fraction of discharge (error bars indicate $\pm 1 S E$, light grey circles indicate linearly interpolated event water fractions when discharge isotope measurements are missing (second panel from the bottom), as well as soil moisture at the grassland site (light green) and forest site (dark green, bottom panel). 

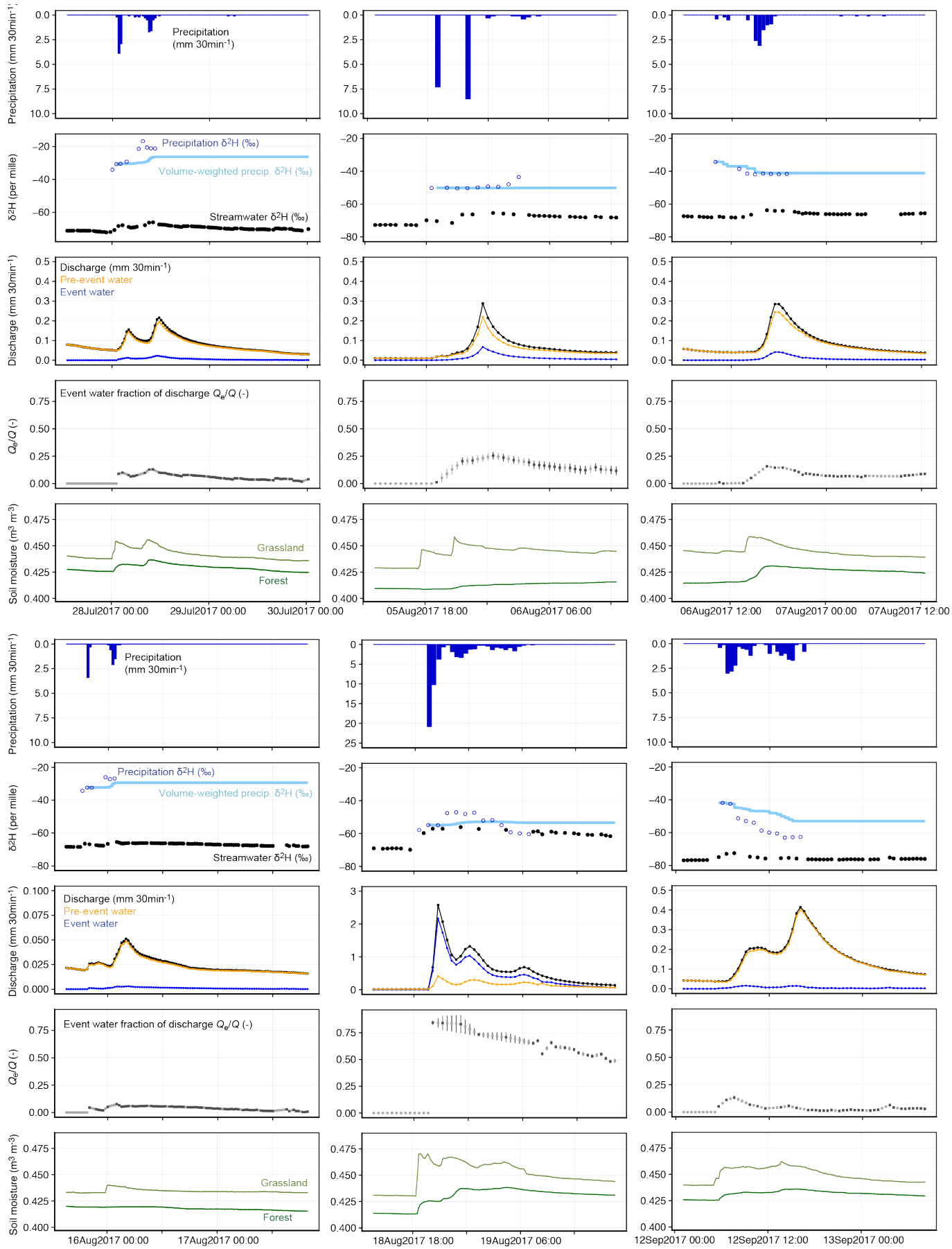

Figure S 3: Time series of storm events between 28Jul2017and 12Sep2017, showing precipitation hyetographs (top panel), deuterium abundance $\left(\delta^{2} H\right)$ in precipitation (with individual measurements in dark blue and incremental weighted means in light blue), and deuterium streamwater (black, second panel from top), discharge hydrograph separated into event and pre-event water (third panel from the top), event water fraction of discharge (error bars indicate $\pm 1 S E$, light grey circles indicate linearly interpolated event water fractions when discharge isotope measurements are missing (second panel from the bottom), as well as soil moisture at the grassland site (light green) and forest site (dark green, bottom panel). 

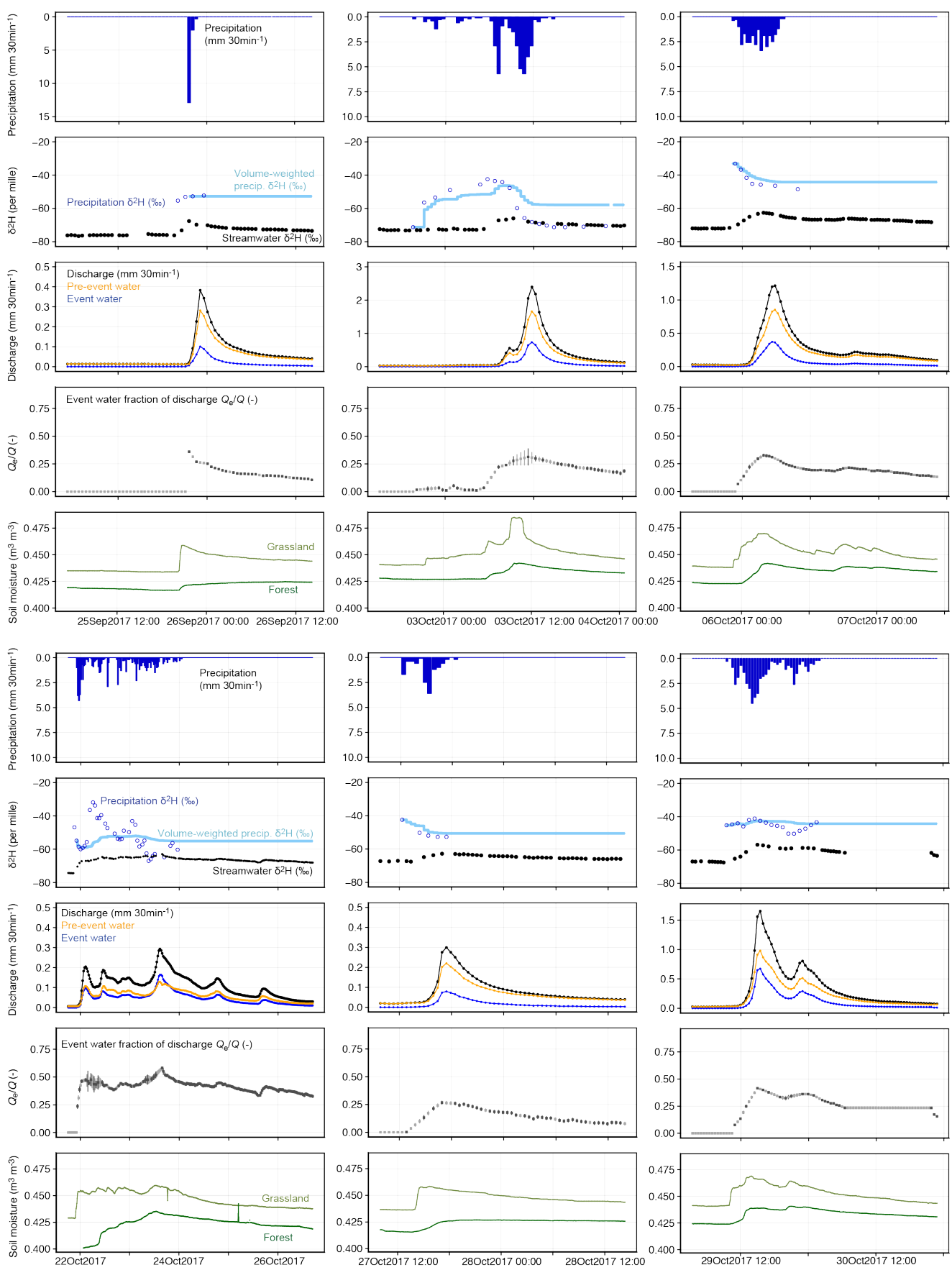

Figure S 4: Time series of storm events between 25Sep2017and 29Oct2017, showing precipitation hyetographs (top panel), deuterium abundance $\left(\delta^{2} \mathrm{H}\right)$ in precipitation (with individual measurements in dark blue and incremental weighted means in light blue), and 5 deuterium streamwater (black, second panel from top), discharge hydrograph separated into event and pre-event water (third panel from the top), event water fraction of discharge (error bars indicate $\pm 1 S E$, light grey circles indicate linearly interpolated event water fractions when discharge isotope measurements are missing (second panel from the bottom), as well as soil moisture at the grassland site (light green) and forest site (dark green, bottom panel). 